\title{
Trends in mathematical modeling of host-pathogen interactions
}

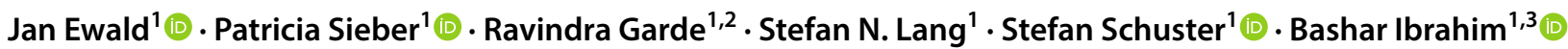

Received: 5 November 2019 / Revised: 5 November 2019 / Accepted: 12 November 2019 / Published online: 27 November 2019

(c) The Author(s) 2019

\begin{abstract}
Pathogenic microorganisms entail enormous problems for humans, livestock, and crop plants. A better understanding of the different infection strategies of the pathogens enables us to derive optimal treatments to mitigate infectious diseases or develop vaccinations preventing the occurrence of infections altogether. In this review, we highlight the current trends in mathematical modeling approaches and related methods used for understanding host-pathogen interactions. Since these interactions can be described on vastly different temporal and spatial scales as well as abstraction levels, a variety of computational and mathematical approaches are presented. Particular emphasis is placed on dynamic optimization, game theory, and spatial modeling, as they are attracting more and more interest in systems biology. Furthermore, these approaches are often combined to illuminate the complexities of the interactions between pathogens and their host. We also discuss the phenomena of molecular mimicry and crypsis as well as the interplay between defense and counter defense. As a conclusion, we provide an overview of method characteristics to assist non-experts in their decision for modeling approaches and interdisciplinary understanding.
\end{abstract}

Keywords Dynamic optimization $\cdot$ Game theory $\cdot$ Agent-based modeling $\cdot$ Equation-based modeling $\cdot$ Host-pathogen interactions · Crypsis

\section{Introduction}

Pathogenic organisms have been an immense burden since the beginning of civilization and still continue to afflict us through deadly infectious diseases that affect humans, animals, or plants. Even today, in a world where we have developed advanced antibiotics designed to specifically target and suppress pathogens, microbial pathogens continue to be a leading cause of disease that causes major loss of human lives, crops, and livestock. For example, human infections

Stefan Schuster

stefan.schu@uni-jena.de

$\triangle$ Bashar Ibrahim

bashar.ibrahim@uni-jena.de

1 Matthias Schleiden Institute, Bioinformatics, Friedrich Schiller University Jena, Ernst-Abbe-Platz 2, 07743 Jena, Germany

2 Max Planck Institute for Chemical Ecology, Hans-Knöll-Str. 8, 07745 Jena, Germany

3 Centre for Applied Mathematics and Bioinformatics, Gulf University for Science and Technology, 32093 Hawally, Kuwait are estimated to cause over 8 million deaths in 2019 [90] and plant pathogens are responsible for a 20-30\% yield loss of major food crops [108]. While many infectious diseases have practically been eradicated, new problems such as antibiotics resistance have emerged, which considerably increase the clinical and economical burden [131]. Besides bacteria and viruses, pathogenic fungi such as Candida albicans or Aspergillus fumigatus are an underestimated threat [18].

In conjunction with experimental and clinical investigations, computational and mathematical approaches have turned out to be highly valuable in understanding and diagnosing host-pathogen interactions (HPI) and devising optimal therapies [9, 30, 109]. Furthermore, computational modeling of biological conditions can save time and money compared to wet lab experiments, and can simulate certain processes that are hardly realizable in experiment [21].

Computational approaches have led to several success stories already. They have been very helpful in predicting, assessing, and controlling epidemics [117]. To that end, epidemiological models are used. On the micro host-pathogen level considered in this review, for example, the metabolism of Trypanosoma brucei, the causative agent of the sleeping disease, was analyzed by mathematical modeling. That led to 
the prediction that glucose transport is a promising drug target without collateral damage to the host [3], as has indeed been confirmed in experiment [51].

In the present review, we give an overview of computational approaches describing HPI, with a special reference to dynamic optimization, evolutionary game theory, and the modeling of spatial phenomena. The following aspects are covered, in this order, in the sections "Dynamics of infection and optimization", "Costs and benefits", "Molecular mimicry and crypsis", "Spatial properties", and "Defense, counter defense and counter-counter defense": the dynamics of infection, costs, and benefits of HPI as described by game theory, molecular crypsis of pathogens, spatial properties, and the levels of defense interactions. As the name suggests, dynamic optimization is based on optimality principles [38], which are in line with Darwin's concept of 'survival of the fittest'. Also game theory is based on that concept, the idea being that each 'player' evolves such as to maximize its fitness $[57,59]$. However, as the counterparts in the game interfere, they may hinder each other to attain optimal states.

The question arises why various computational methods are applied. In some cases, simple optimization (rather than game theory) is sufficient, notably if the tendencies of the 'players' (e.g., organisms) to increase their fitness are not in conflict with each other. Many processes can be described by alternative methods. For example, the interplay of alleles can often be described both by population genetics and game theory [60]. It depends on the aim of study which method is most suitable [26] or may be, to some extent, a matter of taste. This will be discussed in more detail in the section "Conclusion and outlook".

\section{Dynamics of infection and optimization}

During the interaction of host and pathogens, speed and timing are crucial for both to survive. Therefore, the method of dynamic optimization is valuable to describe and understand infection processes. Originating from engineering, dynamic optimization, also called optimal control (closed-loop problems), describes biological systems by an ordinary differential equation (ODE) system whose behavior is influenced by control or decision variables [5, 80]. On a molecular level, host and pathogenic cells, for example, use enzyme levels (as changed by gene expression, etc.) to control the flux in metabolic pathways and respond to environmental changes [37, 38, 73]. In models of HPI, often cells and their behaviors like proliferation or recruitment are viewed as control variables. These control variables are then optimized with regard to an objective function to get a time-optimal strategy controlling the behavior of the biological system (see Fig. 1). While there are many models of infections and HPI using ODEs [35], dynamic optimization is less frequently used to study host-pathogen systems.

Commonly, dynamic optimization is used for the analysis of transmission dynamics and epidemiology by extending the popular Susceptible-Infected-Recovered models [35, 113]. Although these models are valuable to obtain strategies controlling the spread of infections, we want to emphasize, in this review, dynamic optimization models describing infection processes and HPI within the host.

Dynamic optimization problems focusing on withinhost dynamics are formulated in two main fields of application. In the first field, parts of the immune response

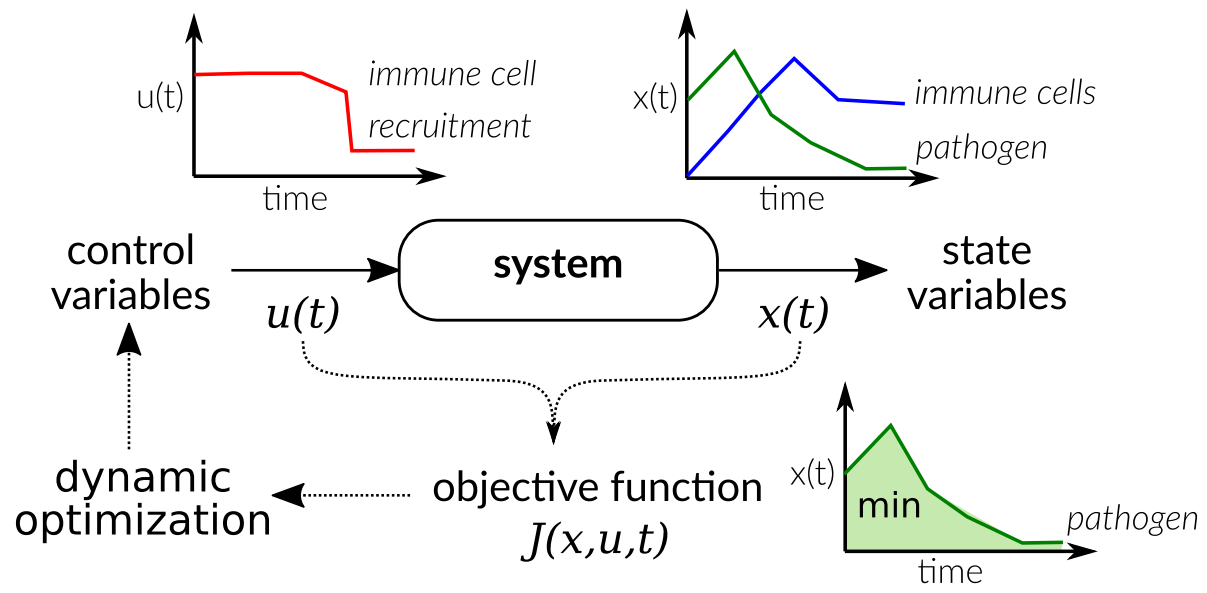

Fig. 1 Basic concept of dynamic optimization illustrated by a simple host-pathogen system. A system, in the depicted example the growth of pathogens (green) and their phagocytosis by immune cells (blue), is described by state variables $[x(t)]$. The behavior of the system is influenced by control variables $[u(t)]$, for example the recruitment of immune cells (red) to combat the pathogen. The control variable is optimized with regard to an objective function to find, for example, the optimal time course of recruitment of immune cells to minimize the pathogen load. Such time courses often show a switch-like behavior between upper and lower bounds (the so-called bang-bang control) 
itself are described by control variables. Defense strategies against pathogens are determined which maximize the health of the host and minimize the number of pathogens (see the example in Fig. 1). This idea was applied already more than 40 years ago by Perelson and colleagues in a series of publications studying the optimization of proliferation and differentiation of lymphocytes [98-100]. Later, Shudo and Iwasa postulated two dynamic optimization problems for these processes. First, they investigated the dynamic optimization of different defense options of the host, which differ in their ability to inhibit the growth of the pathogen, damage to host cells, or their time delay [115]. In a subsequent work, the immune response was viewed as an optimizing problem where the number and proliferation of immune cells is optimized while controlling pathogenic cells and minimizing tissue damage by immune cells [116].

Recently, Dühring et al. [29] applied dynamic optimization to the question of macrophage replication during their interaction with $C$. albicans and could provide evidence that immediate phagocytosis of fungal cells is mostly preferred over replication to increase immune cell number first. This supports the recommendation 'hit hard and hit fast' of Paul Ehrlich stated a hundred years ago about the combat against microbes [34]. This characteristic is disclosed by nearly all dynamic optimization models of the immune response and is generically illustrated in Fig. 1. It shows that pathogens are best controlled by a quick and strong response. The increase in immune cells is then due to (fast) recruitment rather than (slow) proliferation. Mathematically, this can be easily explained by the non-linear exponential growth of pathogens. Due to this fast growth, also the effort to clear the pathogen would rise exponentially with time if applied later.

Empirically, the strategy 'hit hard and hit fast' has been proven to be of advantage for several reasons. First, a high average steady-state concentration of antimicrobial drugs enables a shorter antibiotic treatment and prevents generation of resistant strains [84]. Second, antibiotics are administered with a higher initial dose, the so-called loading dose. The loading dose is given to rapidly achieve an effective drug concentration in the blood and tissues, and underlines the importance of a quick response [86]. Additionally, a fast and strong response reduces peak microbial load and, therefore, lowers the risk of a septic shock [76].

Similar ideas are investigated in the second main field of models using dynamic optimization. Here, Stengel et al. $[121,122]$ introduced dynamic models to determine timeoptimal medical treatment strategies for an arbitrary pathogen. In that and subsequent work, the idea is to determine a time-optimal treatment schedule of using antibiotics, antivirals, etc., and also the optimal choice of different options, notably antimicrobials, healing factors, or immune enhancements [120-122].
Studies determining the time-optimal dosing strategies can help to reduce host damage, e.g., by sparing the commensal microbiome during the antibiotic treatment [97], while keeping a fast and strong intervention against pathogens. More recently, the modulation of inflammation during infection was investigated by multiple authors, who determined optimal dosing schedules of mediators for pro- or anti-inflammation [6, 24, 138].

All of the models discussed above focus on the optimization of the host's health status. However, dynamic optimization can also be applied to understand the evolution of virulence as shown by Ebert and Weisser [31], who determined the optimal time point to kill the host from the viewpoint of a parasite.

In addition to the stand-alone models, dynamic optimization has emerged as a valuable approach in multi-scale modeling to complement other models with different scale or scope [109]. An example is the model of Chen et al. [20], which extends the idea of optimal treatment scheduling proposed by Stengel et al. [121]. This leads to a dynamic gametheoretical model. That work as well as the above-mentioned publication on the dynamics of macrophage replication and C. albicans evasion strategies [29] show that the combination of dynamic optimization and game theory is valuable to gain insights into the dynamics and evolutionary aspects of HPI. Moreover, aggregation of dynamic optimization with agent-based models describing the control of population dynamics in a 2D (e.g., on a lung epithelium) or 3D environment illustrates the potential for multi-scale models of infections using both approaches [2, 39].

Despite the presented successful application of dynamic optimization to HPI, an even closer integration with experimental data as well as more concrete suggestions for clinical applications are worthwhile. A similar point was made by Eftimie et al. [33], highlighting optimal control as a valuable mathematical approach in immunology, which can improve clinical intervention strategies. A role model is the application of optimal control to the management of diabetes, where dose regime optimization is realized not only in silico but also in clinical trials $[43,83]$.

\section{Costs and benefits}

In the evolution of HPI, the costs and benefits of the employed strategies determine the fitness of both host and pathogen. As a mathematical description, game theory [46, 57] has proven to be of great value, and describes the difference between benefits and costs of strategies in a payoff matrix [11, 59, 127]. Based on this, stable solutions (sets of strategies) can be calculated, which are called Nash equilibria and are predictions for evolutionarily favorable strategies. The generalization of that concept is called evolutionarily 
stable strategy (ESS) [46, 57]. Game theory can provide insights into the evolution of interaction phenomena like the persistence of a pathogen. Nevertheless, most models are small and comprehensible, and the solutions are easily obtainable. While the payoffs are often difficult to quantify exactly, it is often sufficient to define the order relations between them to determine the Nash equilibria. A drawback of the approach is that the high level of abstraction can hamper a direct experimental validation.

In the following, we summarize different types of games and show their applications to HPIs. Typically, host and pathogens are considered as players with different strategies and unequal payoffs resulting in asymmetric games (see Table 1). Most models in game theory consider two possible strategies for each player. Accordingly, many game-theoretical models describing HPI are asymmetric two-player two-strategy games.

Intuitive strategies for host and pathogenic cells are either aggressive or non-aggressive against the other cell type. This is also referred to as attack and silence or as 'killer' and 'diplomat', respectively (see Tables 1 and 2). As one of the first game-theoretical models of HPI, Renaud and de Meeüs [106] found that there is always the ESS in which pathogen and host are aggressive (attack against host and suppression of pathogen, respectively). Only for some parameter values, the 'diplomat' strategy is a solution as well. That is, 'war' is always a stable situation, while 'peace' is an additional stable situation (implying bistability) only under certain conditions, notably if $T_{\mathrm{P}}<R_{\mathrm{P}}$ and $S_{\mathrm{H}}<R_{\mathrm{H}}$ in Table 2 (notation according to Prisoner's Dilemma: $R$, Reward for mutual cooperation; $T$, Temptation to defect; $S$, Sucker's payoff; $P$, Punishment for mutual defection). This implies that the cost of virulence (pathogen) and the cost of resistance (host) exceed the loss due to hosting the pathogen.
Table 2 Example of a general payoff matrix for games between host and pathogen cells. Adapted from [106] with notation according to Prisoner's Dilemma: $R$ Reward for mutual cooperation, $T$ Temptation to defect, $S$ Sucker's payoff, and $P$ Punishment for mutual defection

\begin{tabular}{c|cc|cc}
\hline \multirow{2}{*}{ Pathogen } & \multicolumn{2}{|c|}{ Killer } & \multicolumn{2}{c}{ Diplomat } \\
\hline Killer & $P_{P}$ & $P_{H}$ & $T_{P}$ & $T_{H}$ \\
\hline Diplomat & $S_{P}$ & $S_{H}$ & $R_{P}$ & $R_{H}$
\end{tabular}

Other asymmetric models focus on intracellular and persistent bacteria and gain insights into evolutionary advantages of attenuated virulence [124] or localization [36] as strategies of pathogenic bacteria. The opportunistic fungal pathogen $C$. albicans aroused attention due to its medical relevance and morphological switch from yeast-like to filamentous growth during infection. Interestingly, this was first studied by symmetric games (which are easier to analyze) between fungal cells, with the immune cells being considered as a constant environment [61, 128] (see Table 1). More recent studies, however, describe asymmetric games between host and pathogenic fungal cells and focus on certain experimental aspects like expression of transcription factors [127] for filamentous growth or evasion strategies resulting in non-lytic expulsion [29].

In addition, symmetric games of pathogens competing within a host are described by continuous strategies and enable Nash equilibria to be calculated and predictions for optimal growth rates [17] or toxin production of pathogens [95] to be made. The latter is also described as an N-player game with two strategies for producing a host cell wall toxin or to cheat and results in a Volunteer's Dilemma [95]. On the other side, games between hosts facing pathogens can also be modeled by N-player games. For example, some models describe the overuse of antibiotics resulting in a Tragedy of
Table 1 Types of host-pathogen games

\begin{tabular}{|c|c|c|}
\hline & asymmetric & symmetric \\
\hline \multicolumn{3}{|l|}{2 players } \\
\hline $\begin{array}{c}2 \\
\text { strategies }\end{array}$ & $\begin{array}{l}\text { a) attack } \\
\text { b) silence }\end{array}$ & $\begin{array}{l}\text { a) antibiotics } \\
\text { b) no antibiotics }\end{array}$ \\
\hline $\begin{array}{c}>2 \\
\text { players }\end{array}$ & vs. $\sum_{\text {Commensal }}^{\text {Patho }}$ & \\
\hline $\begin{array}{c}>2 \\
\text { strategies }\end{array}$ & & $\begin{array}{l}\text { a) pathogen } \\
\text { b) sensitive commensal } \\
\text { c) tolerant commensal }\end{array}$ \\
\hline $\begin{array}{l}\text { continuous } \\
\text { strategy }\end{array}$ & & growth rate \\
\hline
\end{tabular}


the Commons $[41,105]$. This can be used to draw conclusions for the prescription of antibiotics.

To better explain the biodiversity of pathogens or the host's microbiome, models with three players and/or three strategies are defined. For example, Wu and Ross [135] investigated the asymmetric game of two types of commensals (sensitive or non-sensitive to antibiotics) and a pathogenic bacterial species. That model explains the common observation that supporting commensal bacteria can alone be effective to eradicate pathogenic bacteria in the gut of a host. Another interesting perspective was investigated by Morozov and Best [87], who used game theory to model the interplay of predators, prey, and pathogens infecting the prey.

Furthermore, an emerging trend in modeling HPI is to extend and represent game-theoretical models with graphs and lattices adding spatial and stochastic characteristics. With these multi-scale models using methods of agent-based modeling, the invasion of bacteria [72] and lung infections by A. fumigatus have been modeled [104].

\section{Molecular mimicry and crypsis}

An important aspect in HPI is the multicellularity of the host. Moreover, hosts usually harbor not only their own cells but also non-self cells. As the specialized compartments are highly interdependent, hosts usually provide common goods to all of their cells, for example, using the blood stream. This strategy is very effective, but susceptible to parasitism or the Tragedy of the Commons. To prevent this, hosts developed mechanisms for discrimination of damaged self cells as well as parasitic (or pathogenic) non-self cells from intact self cells and beneficial non-self cells and for restricting any nonself cells using the common goods. Beneficial non-self cells are hosted on the interface to the outside, e.g., the skin or the lumen of the gut, providing mutualistic protection and nutrition $[28,45,110]$.

Protection inside the host is ensured by specialized cell lineages and their interactions, forming the immune system. In vertebrates, this system can be divided into innate immunity, detecting generic characteristics of non-self cells and tagging these cells, and adaptive immunity, which responds specifically to particular pathogens [91]. Both mechanisms rely on signals which, after interpretation by possibly several parts of the immune system, lead to an immune response. For example, vertebrates have developed a system in which each cell entering the blood stream is tagged non-specifically with complement factors like complement component $3 \mathrm{~b}$ (C3b) [93]. Those tagging complement factors are inactivated specifically on intact self cells by inhibiting complement factors like factor $\mathrm{H}$. If a phagocyte detects sufficient amounts of tagging complement factors on the surface of a cell, it will remove the cell. In contrast, if pathogens are able to remove the signal from their surfaces themselves, they could avoid attack.

To model HPI, it is thus important that the host has to classify the player types based on signals prior to interaction (e.g., self vs. non-self) to decide the optimal response strategy (e.g., attack or 'diplomat' behavior [106]). This discrimination is prone to errors, since individuals imitating self-signals (mimicry) or preventing non-self-signals (crypsis) could dupe the host into using a non-desirable strategy. In HPI, both mimicry (e.g., resemblance of viruses to self-antigens) and crypsis (e.g., complement evasion by fungi and bacteria) occur. Molecular crypsis is achieved by several pathogenic fungi such as $C$. albicans and bacteria in that they are able to bind human factor $\mathrm{H}$ or other complement regulators on their surface. In this context, not only the evasion of pathogens is problematic but, because of the indistinguishability between self and non-self, also autoreactivity may arise [136].

Since mimicry and crypsis may occur whenever there is signal perception or communication involved, the type of mathematical model used has to be chosen depending on the concrete modeled system. Most commonly, theoretical descriptions distinguish between three entities: a model, a dupe, and a mimic [58]. Note the double meaning of the word 'model' here: It refers to the mathematical description and to the template imitated by the mimic. The dupe learns the model based on the presence or absence of one or more characterizing signals. Due to noise in the perception system and diversity of the signal within the species, the perceived signal is usually modeled approximately using a Gaussian distribution [58, 133]. The closer the mimic resembles the model, the more the distributions will overlap, making it more difficult for the dupe to distinguish them. In agentbased models, signals could be inferred from explicit traits.

Given the signals, it is next important to define interactions between the three entities and the respective consequences on their fitness. This includes, for example, whether the model is attractive or should be avoided by the dupe. Also, the costs of erroneous classification as false positives or false negatives are very relevant. The classification task can then be modeled using any classifier, for example optimization of a classification threshold with respect to a fitness function or training of a neural network based on the previous interactions.

Perfect resemblance of the model signals by the mimic is possible in principle, no matter how sophisticated the classifier is, but may never be achieved. This is because the dupe and mimic constantly adapt and there is always an arms race in identifying and hiding, being attacked and evading [77, 81]. For example, in the case of complement evasion, most microorganisms are able to remove complement factors (which would identify them as pathogens) by utilizing the 
host-protecting complement factor $\mathrm{H}$ [137]. The host can make this adaption harder using polymorphous protection molecules, i.e., two different alleles of factor $\mathrm{H}$ and factor $\mathrm{H}$-like molecules in addition.

In the case of adaptive immunity, pathogens could potentially mimic the serotype of self cells perfectly. Again, polymorphisms make adaption for pathogens more difficult. For example, the AB0 blood group polymorphism may protect against molecular mimicry (here on a population level), as pathogens mimicking type A antigens will not be able to survive in type B hosts [22]. Such mechanisms use the fact that pathogens usually transmit via several hosts, while host cells are not meant to migrate between hosts. On the other hand, the presence of mimicking pathogens will sensitize opposing types against each other, with great influence in transplantology. For example, a pathogen mimicking type A will induce anti-A antibodies in types B and 0 .

While mimicry has been intensely analyzed by mathematical modeling in the case of higher animals $[58,119]$, only a few modeling studies on camouflage by microorganisms have been published so far. The term 'molecular mimicry' had been introduced by Damian in 1964 [22]. Recently, Lang et al. used signaling theory and ODEs to describe and analyze the attack decision by the host in the case of molecular crypsis [77]. That decision is non-trivial, because the two criteria of minimizing false negatives (foreign cells erroneously recognized as self) and minimizing false positives (own cells erroneously recognized as foreign) cannot be met simultaneously. The model identified relative pathogen abundance to be the predominant factor influencing successful molecular crypsis. If pathogen cells attain a quantitative advantage over host cells, even autoreactivity may occur. An alternative approach uses game theory [60]. It can nicely describe the tradeoff between auto-immunity (e.g., age-dependent macula degeneration) and susceptibility to bacterial infections as well as the dimorphism of the factor $\mathrm{H}$ gene.

By plausibility arguments, the qualitative dependence of fitness on the investment into crypsis can easily be derived (Fig. 2). At low values of investment, fitness decreases practically linearly due to costs involved and because there is no benefit yet when the extent of crypsis is low. For example, a snake being purely black or purely red has achieved half of the coloring of poisonous snakes having black and red stripes but does not practically have any mimicry effect. Thus, there is a local maximum at zero investment. The global maximum is reached at a certain high investment if mimicry is efficient enough. A curve shape similar to that shown in Fig. 2 could indeed be obtained by numerical simulation of mimicry by $C$. albicans (Fig. 5 in [77]). In Fig. 2, the curve goes down beyond the global maximum based on the plausible

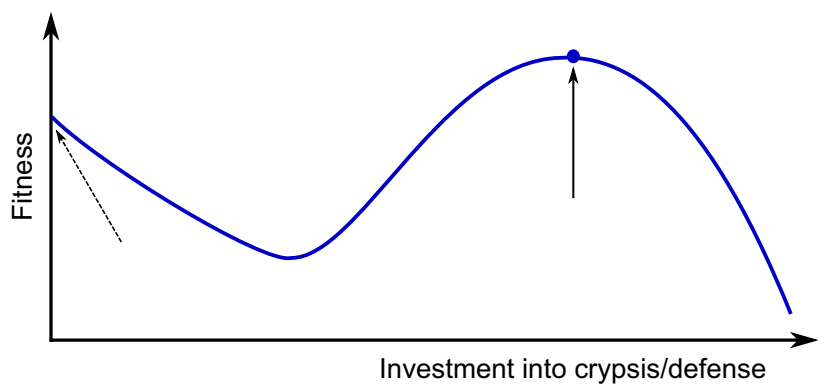

Fig. 2 Qualitative dependence of fitness on the investment into crypsis or defense. The dashed arrow indicates the local maximum; solid arrow and blue dot: global maximum. The local maximum can be reached without additional investment. With a low amount of investment, the costs are higher than the benefit, which results in a decrease of fitness. Only a high investment is effective and leads to the global maximum. For further explanations, see section "Molecular mimicry and crypsis" and Lang et al. [77]

assumption that an investment that is too high, takes away too much from the resources. It is an interesting open question how the global maximum could be attained in evolution as it is difficult to explain by small-scale mutations (micro-evolution). A possible solution is horizontal gene transfer, although this would not explain how the trait emerged in the first place.

\section{Spatial properties}

There are two main approaches commonly used to model and simulate spatio-temporal dynamics: (i) a top-down equation-based modeling (EBM) approach and (ii) a bottomup agent-based modeling (ABM) approach (also known as individual-based modeling). Each of these approaches has its own weaknesses and strengths depending on the questions to be answered and the hypotheses to be validated. The most widely used top-down approaches are differential equations-based: either ordinary (ODEs), partial (PDEs), stochastic (SDEs), or delay differential equations (DDEs). Another type is formed by discrete models such as difference equations.

ODE modeling is used, for example, to describe metabolic processes in pathogens [3]. The ODE or discrete models generally ignore the topology, while PDEs consider the spatial distribution to some extent (e.g., diffusion but not geometry of molecules). EBM does not deal with single entities but rather with describing populations by estimating the mean behavior at a macroscopic level [3, 62, 64, 69, 70, 88]. It uses a set of equations that are based on the relationships among observables. Solving these equations particularly in higher dimensions is based on numerical techniques, which reproduce the dynamics of variables (population densities, concentrations, etc.) over time. EBM has been widely 
employed to study the dynamics of bacteria [19, 88], fungi [79], viruses (for review, see [8, 12, 25, 52, 101, 139]), and has also been applied to other areas such as cell division mechanisms (e.g., [53, 63, 65-67, 129, 130]).

The bottom-up approach, including ABM or cellular automata (CA) such as the famous 'Game of life' [42], works at a microscopic level $[13,14,118]$. Both ABM and $\mathrm{CA}$ are suitable methods to simulate the behavior of a system in a spatio-temporal manner. However, CA are gridbased and do not allow a free movement in space as the (usually) lattice-free ABM does [14, 21, 96, 118]. Thus, ABM can be considered as an extension or generalization of $\mathrm{CA}$ $[26,96]$. In the unconventional ABM approach, individual independent agents are defined (such as fungal, bacterial, or human cells) with interaction rules [7, 13, 21, 44, 96, 118]. Individual interactions (usually between nearest neighbors) are simulated rather than global conditions [7, 21, 30, 114].

The behavior of the complete system arises from all local behaviors of the entities involved. This can lead to emergent properties of the system, which are not immediately seen from the local interactions. Even with simple rules, very complex spatio-temporal patterns can arise, as can already be seen in the 'Game of Life'. By ABM, the dynamics of different agents within a (complex) system can be analyzed and the impact of different initial settings can be investigated over time [7, 13, 21, 44, 118]. These bottom-up approaches are usually easier to implement than top-down approaches, because it is more comprehensible to describe the behavior of each individual agent than that of the whole system. Also, it can be adapted more flexibly to changing conditions and stochastic factors can easily be included in the rules $[7,13,26,96,118]$. A further advantage is that the results can be visualized nicely, for example, in colorful videos. Thus, ABM is a very powerful method to describe HPI and becomes increasingly important for the scientific community.

The power of $A B M$ has its prize: Depending on the size of the system, ABM can be significantly more computationally expensive compared to EBM. Furthermore, ABM is often a stochastic rather than deterministic approach [7, $44,96,114,118]$. Consequently, it is not sufficient to run a simulation only once, but multiple simulations are required. The rules of the agents have to be realistic and can be gained from experiments, which become more and more available. However, this is not possible for all parameters and can lead to unknown values. Parameter estimation might be time consuming and may change the model outcome dramatically $[26,30,118]$. Taken together, ABM allows modeling with a higher spatial resolution over a longer time period compared to EBM, but it requires more detailed information about the system of interest and it is computationally more expensive. Without detailed knowledge, it might be too abstract for a certain application or may lack complex interaction mechanisms [96].

ABM has been applied across a wide range of disciplines including cell biology, population dynamics, epidemiology, and immunology [14, 21, 96, 117]. Many agent-based frameworks were developed (for review, see [1, 7, 15, 118]). Some famous examples are iDynoMics [78], MASON [82], NetLogo [132], and BSim [48, 85] which are user-friendly and can also be used by non-modelers. In addition, frameworkindependent implementations are used for ABM to simulate HPI [23, 102, 123]. NetLogo [21, 132] is one example for a modeling framework with its own programming language, with 'turtles' that represent agents and 'patches' that represent points in the simulation space. It can be applied easily to many different questions and has widely been utilized for HPI modeling [27, 89, 96, 112, 125]. NetLogo allows users to write their own extensions. However, it cannot incorporate formal rule-based languages such as BNGL (BioNetGen language) [10] or Kappa [16], nor molecular structure and geometry (for details, see [47, 49, 50, 54, 55, 71, 126]). In addition, it is challenging to handle very large network models or very low concentrations of agents with stochastic rules $[68,74,75]$.

In the following, we provide a toy example using NetLogo to illustrate the ABM approach (see Fig. 3). Macrophages in interaction with a pathogenic species are modeled in a defined space. When macrophages recognize a pathogen, they try to phagocytose it. If the pathogen has too little energy to resist, it is taken up by the macrophage. In the implemented model (see Fig. 3), the user can define certain values for the number of individuals per species, its energy resources, and speed of movement. With these chosen parameter settings, the model can be initialized and run, and the movement of the agents can be visualized during the simulation. The time behavior of the energy level and number of species is monitored in different plots (see Fig. 3b). The simulated behavior can be extracted and processed or compared in further steps. In our example, it shows that the process of HPI is energy-consuming for both macrophages and pathogens. It depends on the number of macrophages how well pathogens can be phagocytosed.

Tokarski et al. implemented a related model using NetLogo, which shows that a communication between the immune cells (neutrophils in that case) is beneficial during HPI [125]. That model also indicated that hunting by neutrophils in clusters (like wolves in a wolfpack) is only beneficial if also the fungal pathogens occur in clusters. Otherwise, too much of the area remains uncovered by the neutrophils.

In another ABM, the movement of human alveolar macrophages searching A. fumigatus spores on the lung epithelium was modeled [102]. The model predicted that randomly migrating macrophages fail to find the spore before the start of germination, whereas guidance by chemotactic signals 

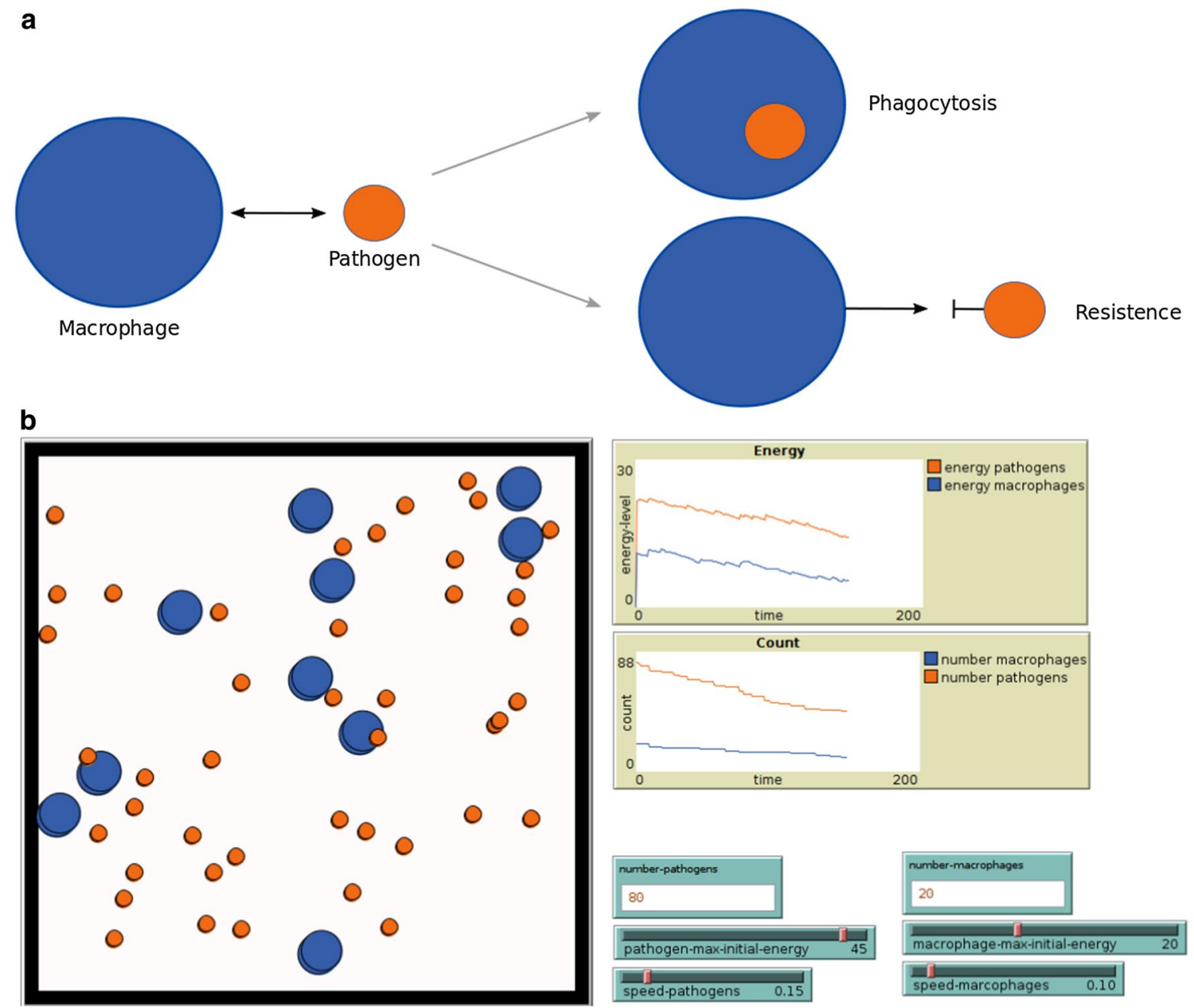

Fig. 3 Toy example of HPI formalized as an agent-based model. a Individuals of macrophages (blue) phagocytose the pathogens (orange) when they are touching each other and the latter have too

little energy to resist. b Screenshot of the simulation using NetLogo. During the simulation, the energy of macrophages and pathogens and the number of individuals are plotted

enables a safe and successful discovery of the pathogen in time (for an extended model, see [9, 103]). A further study about inhaled $A$. fumigatus spores considers a broader view of the lung tissue and immune cells [89]. This model focuses on the role of iron for both fungal and host cells, and shows that iron is a critical factor for fungal growth [89].

Bacterial infection has been investigated for Pseudomonas aeruginosa in the gut. It has been shown that an unbalanced gut flora can lead to increased virulence of $P$. aeruginosa and ABM was used to understand mechanisms of HPI [112, 123]. For example, Stern et al. used this approach to analyze the binding of the bacterium to the epithelium and suggest

that integrin-laminin associations play an important role for bacterial virulence [123]. Furthermore, the infection of the intestinal tract by Clostridium difficile has been described by $\mathrm{ABM}$ to observe the efficiency of treatments against this bacterium [96]. The inflammation process in humans was in general formulated by ABM by Dong et al. They show that ABM is very suitable to describe complex systems, and model the host response and observe specific signaling, such as NF- $\kappa \mathrm{B}$ [27]. The NF- $\kappa \mathrm{B}$ pathway was investigated using $\mathrm{ABM}$ in other studies and reviewed by Williams et al. [134]. 


\section{Defense, counter defense, and counter- counter defense}

In many parasitic interactions, the host organisms protect themselves by toxic compounds (defense chemicals) or other mechanisms such as mechanical barriers (e.g., skin), fever, $\mathrm{pH}$ changes, etc. Many of the pathogens, in turn, produce enzymes degrading the defense chemicals, which can be considered as a counter defense (a.k.a. evasion mechanisms). Examples are provided by the HPI between the human innate immune system and the pathogenic fungus $C$. albicans [30]. For example, immune cells produce various reactive oxygen species (ROS). C. albicans and many other pathogens, in turn, can detoxify the ROS by superoxide dismutases (SODs) [40]. All intracellular pathogens, like Plasmodium sp., also deflect defense to escape the humoral immunity of the host.

Interesting analogs can be found in plants. For example, the tomato plant produces tomatine to defend itself against the tomato-wilt fungus Fusarium oxysporum f. sp. lycopersici [94]. Tomatin is a glycoalkaloid with antimicrobial and insectidical properties. As a counter defense, the fungus produces tomatinase [94]. Another similar example is the oat plant which produces avenacin, a saponin which is mainly produced in the roots and provides defense against the fungal pathogen Gaeumannomyces graminis var. avenae by binding to sterols in the fungal cell membrane. The fungus responds by producing avenacinase, an enzyme that degrades avenacin [92].

Counter defenses represent the secondary phase of the HPI, i.e., they depend on the primary phase to be of any use at all. Thus, it might be risky to invest resources towards a robust counter defense without having a prior knowledge about the likelihood that the host will exhibit a formidable defense. Game theory and, more generally, operations research are powerful tools that can help to assess the costs and benefits of such interactions and the coevolution of host and parasite.

Clearly, the dependence of fitness on the investment into defense (or higher levels such as counter defense) is similar to that shown in Fig. 2. Again, a low extent of defense is practically useless, while it inflicts costs. For example, a toxin at low concentrations has (nearly) no effect. An

Table 3 Payoff matrix of a game between host and parasite with defense and counter defense. Payoffs are such that the cost of defense is twice as much as the damage. The Nash equilibrium is underlined

\begin{tabular}{c|cc|cc}
\hline \multirow{2}{*}{ Host } & \multicolumn{2}{|c|}{ Defense } & \multicolumn{2}{c}{ No response } \\
\hline Counter defense & -2 & -6 & -2 & -2 \\
\hline No response & 0 & -4 & $\underline{2}$ & $\underline{-2}$ \\
\hline
\end{tabular}

interesting question is under which conditions it pays, during evolution, to establish a counter-counter defense rather than to intensify or widen an existing defense [30,111].

Let us consider a game among a host and a pathogen where the cost of defense and counter defense is four units of resources. We assume that the pathogen has already played attack, and thus, the host starts the game with a damage of two units, i.e., the cost is twice as much as the damage. The pathogen has invaded the host successfully and, therefore, enjoys a reward of two units. The host can either choose to respond with a defense or choose to do nothing. The pathogen on the other hand can choose to do nothing or escalate the interaction to a further level by a counter defense. The payoffs for each of these strategies are summarized in Table 3.

In the scenario where both players choose the non-peaceful strategy, i.e., the host chooses to defend itself and the pathogen responds with a counter defense, both incur a cost of four units. In the absence of defense, counter defense only costs the pathogen four units of resources. In a scenario where the pathogen does not respond to a defense of the host, the host incurs a cost of four units. When both players choose to show no response, the host has a damage of two units from the attack, while the pathogen, having invaded the host, enjoys a reward of two units as per assumption. Clearly, choosing the peaceful 'no response' strategy by both players seems the least costly choice. This is also the Nash equilibrium, since neither player has an incentive to change strategy unilaterally.

Let us now consider a scenario where the cost is only one unit, i.e., the damage is twice as expensive as the cost. The resulting payoff matrix is depicted in Table 4 and shows that the host has an advantage in playing defense, since it gives a better payoff than when none of the players show any response. However, the pathogen has an incentive to respond with a counter defense, implying that the host obtains the worst payoff possible. Thus, the host is likely to choose no response again, leading to a cycle. There is no pure Nash equilibrium for the game.

The shuffle through the strategies could occur, for example, through gain and loss of function in various genes responsible for (counter)defense. Moreover, it is worth noting that the above approach is based on a discretization to two strategies only on each side. In reality, the amount of the

Table 4 Payoff matrix of the defense/ counter defense game as in Table 3 but with the cost being half of the damage. This game possesses no pure Nash equilibrium

\begin{tabular}{c|cc|cc}
\hline \multirow{2}{*}{ Pathogen } & \multicolumn{2}{|c|}{ Defense } & \multicolumn{2}{c}{ No response } \\
\hline Counter defense & 1 & -3 & 1 & -2 \\
\hline No response & 0 & -1 & 2 & -2 \\
\hline
\end{tabular}


defense chemical and the counter defense (e.g., degrading enzyme) can be chosen in a continuous way. A mixed Nash equilibrium could then mean that a certain percentage of the full amount is produced.

By the traditional approach of game theory used here, it cannot be decided whether a self-sustained oscillation in time between the two choices arises or the strategies are chosen with a certain probabilities in a stochastic way as in the famous rock-scissors-paper game [46] or only part of the defense/counter defense is realized. One way of answering that question is using ODEs, as was used for modeling the rock-scissors-paper game among bacteria [88].

\section{Conclusion and outlook}

In recent years, mathematical and computational approaches have successfully been applied to study HPI from various angles. In synergy with experimental work, in silico models of HPI led to a better understanding of virulence factors and their evolution, a disentanglement of the complex immune response and suggestions for novel or improved antibiotic treatments.

For the future, we see several trends impacting the modeling of HPI. First, the implementation and simulation of models like agent-based and dynamic optimization models is becoming more and more practicable for non-experts by user-friendly tools such as NetLogo or AMIGO2 (toolbox for dynamic modeling and optimization [4]). The second major trend is the availability and integration of vast biological data from omics approaches and imaging. These data allow dynamic and spatial modeling to be highly quantitative and to reach a better predictive power. A similar goal is pursued by multi-scale modeling, which is an additional important trend in models of HPI.

It is often useful to validate mathematical models by alternative modeling methods and frameworks [91], to verify that the results are not an artifact of the particular framework used. As discussed in detail by Schleicher et al. [109], the combination and integration of different approaches is valuable to exploit the large amount of biological data and complex networks of HPI. A combined approach often improves the applicability and value of mathematical models, as pointed out in Section "Defense, counter defense and counter-counter defense" with respect to game theory and ODEs $[9,19,29,56,88]$. Moreover, game-theoretical approaches can be combined with ABM, dynamic optimization [29], or in the form of games on grids or graphs [107].

Associated with numerous computational approaches, the challenge is to choose the appropriate modeling approach. While there is no definitive answer to the question which modeling approach and method is best to model an HPI, one can base the decision on general method characteristics (see Fig. 4). Important distinctive features of the mainly presented methods from game theory, dynamic optimization, and ABM are their capabilities to resolve system dynamics, spatiality, and stochasticity. This means, for example, that one should consider modeling an HPI by $\mathrm{ABM}$ if interaction partners occur in low numbers (stochasticity) or diffusion of signaling chemicals is important (spatiality). While classical game theory does not resolve dynamics, spatiality, or stochasticity per se, it inherently can model coevolution, since interaction strategies are optimized simultaneously and not independently (as in dynamic optimization) or are fixed (as in ABM). Furthermore, the analysis does not require high computational power like ABM due to its stochastic trait. However, a major drawback is the higher abstraction level of biological systems in game theory, which complicates experimental validation of results in comparison to dynamic optimization and ABM.

As a major strength of all three discussed approaches, we see their accessibility to non-expert users and most importantly their ability to model HPI. These HPI are either directly implemented as interaction events in ABM, interaction kinetics in dynamic optimization, or strategies in game theory.

Prospectively, we see the challenge that models of HPI will be more complex in the future due to the amount of experimental data. This is in contrast to the requirement that models should be comprehensible and minimal, so that a trade-off has to be found. Despite these challenges, efforts in mathematical and computational modeling pay off. For
Fig. 4 Comparison of methods presented and discussed in detail in this review. Classification (fully $\checkmark$, partially $(\checkmark)$, and not capable $X$ ) is based on the original method without special extensions and according to its application in HPI modeling as well as the authors' experience

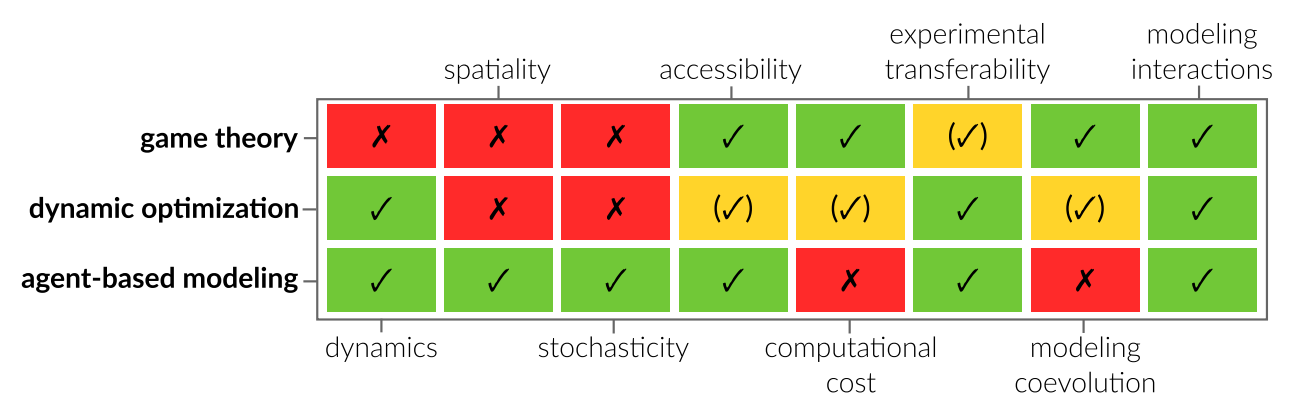


example, spatial modeling disclosed that immune cells benefit from chemotaxic signals to effectively clear pathogens $[102,125]$. These findings now require more experimental work for elucidation. Furthermore, epidemiological modeling of malaria shows the value of combining spatial and dynamic modeling to explore strategies eliminating infectious diseases [32]. Further important examples have been discussed throughout this review. This shows the important value of mathematical modeling in understanding host-pathogen interactions.

Also in the future, modeling of HPI will be an important complement to experimental work to simplify scientific procedures, to reduce (animal) experiments, and to generate hypotheses. We assume that the accessibility of modeling approaches is key to make them applicable for more scientists and recommend the presented approaches which are usable by non-experts. Furthermore, dynamics and spatial observations may have a higher impact over time to describe more complex behavior. This can give rise to new (sub-) approaches to model HPI.

Due to increasing computational power, the limitation of computation (e.g., in ABM and dynamic optimization) will decrease and enable a higher workload and the implementation of large-scale models. Nevertheless, the key in future modeling will be the integration of experimental data and a close collaboration of computational modelers and biological as well as medical experts. This ensures validity of models and decipherment of HPI.

Acknowledgements The authors gratefully acknowledge financial support from the following funding sources: JE and SS are supported by the German Research Foundation (DFG) within the Collaborative Research Center/Transregio 124 FungiNet (Project B1); RG, Max Planck Society through the IMPRS 'Exploration of Ecological Interactions with Molecular and Chemical Techniques'; SL, DFG within the Jena School for Microbial Communication (grant no. DFG-GSC 214/2); PS and BI, DFG within Collaborative Research Center 1127 ChemBioSys (SFB 1127, Project C07). The authors gratefully acknowledge Ines Heiland and Barbara Bakker for fruitful discussions during the International Study Group of Systems Biology (ISGSB) meeting in Norway in 2018. In addition, the authors thank Sybille Dühring and Sebastian Germerodt for helpful discussions.

Author Contributions Idea for the article: SS; literature search: all; data analysis: all; writing — original draft preparation: all; critical revision: SS, BI, JE, and PS

\section{Compliance with ethical standards}

Conflict of interest The authors declare that they have no conflict of interest.

Open Access This article is distributed under the terms of the Creative Commons Attribution 4.0 International License (http://creativeco mmons.org/licenses/by/4.0/), which permits unrestricted use, distribution, and reproduction in any medium, provided you give appropriate credit to the original author(s) and the source, provide a link to the Creative Commons license, and indicate if changes were made.

\section{References}

1. Abar S, Theodoropoulos GK, Lemarinier P, O'Hare GM (2017) Agent based modelling and simulation tools: a review of the state-of-art software. Comput Sci Rev 24:13-33

2. An G, Fitzpatrick B, Christley S, Federico P, Kanarek A, Neilan RM, Oremland M, Salinas R, Laubenbacher R, Lenhart S (2017) Optimization and control of agent-based models in biology: a perspective. Bull Math Biol 79(1):63-87

3. Bakker BM, Michels PA, Opperdoes FR, Westerhoff HV (1999) What controls glycolysis in bloodstream form trypanosoma brucei? J Biol Chem 274(21):14551-14559

4. Balsa-Canto E, Henriques D, Gábor A, Banga JR (2016) AMIGO2, a toolbox for dynamic modeling, optimization and control in systems biology. Bioinformatics 32(21):3357-3359

5. Banga JR (2008) Optimization in computational systems biology. BMC Syst Biol 2(1):47

6. Bara O, Djouadi S, Day J, Lenhart S (2017) Immune therapeutic strategies using optimal controls with 11 and 12 type objectives. Math Biosci 290:9-21

7. Bauer AL, Beauchemin CA, Perelson AS (2009) Agent-based modeling of host-pathogen systems: the successes and challenges. Inf Sci 179(10):1379-1389

8. Beauchemin CA, Handel A (2011) A review of mathematical models of influenza A infections within a host or cell culture: lessons learned and challenges ahead. BMC Public Health 11 Suppl 1:S7

9. Blickensdorf M, Timme S, Figge MT (2019) Comparative assessment of aspergillosis by virtual infection modeling in murine and human lung. Front Immunol 10:142

10. Blinov ML, Faeder JR, Goldstein B, Hlavacek WS (2004) Bionetgen: software for rule-based modeling of signal transduction based on the interactions of molecular domains. Bioinformatics 20(17):3289-3291

11. Bohl K, Hummert S, Werner S, Basanta D, Deutsch A, Schuster S, Theißen G, Schroeter A (2014) Evolutionary game theory: molecules as players. Mol BioSyst 10(12):3066-3074

12. Boianelli A, Nguyen VK, Ebensen T, Schulze K, Wilk E, Sharma N, Stegemann-Koniszewski S, Bruder D, Toapanta FR, Guzman CA, Meyer-Hermann M, Hernandez-Vargas EA (2015) Modeling influenza virus infection: a roadmap for influenza research. Viruses 7(10):5274-5304

13. Bonabeau E (2002) Agent-based modeling: methods and techniques for simulating human systems. Proc Natl Acad Sci USA 99(Suppl 3):7280-7287

14. Bonchev D, Thomas S, Apte A, Kier LB (2010) Cellular automata modelling of biomolecular networks dynamics. SAR QSAR Environ Res 21(1):77-102

15. Bordini RH, Braubach L, Dastani M, Seghrouchni AEF, Gomez-Sanz JJ, Leite J, O’Hare G, Pokahr A, Ricci A (2006) A survey of programming languages and platforms for multiagent systems. Informatica 30(1):33-44

16. Boutillier P, Maasha M, Li X, Medina-Abarca HF, Krivine J, Feret J, Cristescu I, Forbes AG, Fontana W (2018) The Kappa platform for rule-based modeling. Bioinformatics 34(13):i583-i592

17. Bremermann HJ, Pickering J (1983) A game-theoretical model of parasite virulence. J Theor Biol 100(3):411-426 
18. Brown GD, Denning DW, Gow NA, Levitz SM, Netea MG, White TC (2012) Hidden killers: human fungal infections. Sci Transl Med 4(165):165rv13-165rv13

19. Carbo A, Bassaganya-Riera J, Pedragosa M, Viladomiu M, Marathe M, Eubank S, Wendelsdorf K, Bisset K, Hoops S, Deng X, Alam M, Kronsteiner B, Mei Y, Hontecillas R (2013) Predictive computational modeling of the mucosal immune responses during Helicobacter pylori infection. PLoS One 8(9):1-14

20. Chen BS, Chang CH, Chuang YJ (2008) Robust model matching control of immune systems under environmental disturbances: dynamic game approach. J Theor Biol 253(4):824-837

21. Chiacchio F, Pennisi M, Russo G, Motta S, Pappalardo F (2014) Agent-based modeling of the immune system: NetLogo, a promising framework. BioMed Res Int 2014:1-6

22. Damian RT (1964) Molecular mimicry: antigen sharing by parasite and host and its consequences. Am Nat 98(900):129-149

23. Dancik GM, Jones DE, Dorman KS (2010) Parameter estimation and sensitivity analysis in an agent-based model of leishmania major infection. J Theor Biol 262(3):398-412

24. Day J, Rubin J, Clermont G (2010) Using nonlinear model predictive control to find optimal therapeutic strategies to modulate inflammation. Math Biosci Eng 7(4):739-763

25. Dobrovolny HM, Reddy MB, Kamal MA, Rayner CR, Beauchemin CA (2013) Assessing mathematical models of influenza infections using features of the immune response. PLoS One 8(2):e57088

26. Doeschl-Wilson AB (2011) The role of mathematical models of host-pathogen interactions for livestock health and production-a review. Animal 5(06):895-910

27. Dong X, Foteinou PT, Calvano SE, Lowry SF, Androulakis IP (2010) Agent-based modeling of endotoxin-induced acute inflammatory response in human blood leukocytes. PLoS One $5(2): 1-13$

28. Douglas AE, Werren JH (2016) Holes in the hologenome: why host-microbe symbioses are not holobionts. mBio 7(2):e02099-15

29. Dühring S, Ewald J, Germerodt S, Kaleta C, Dandekar T, Schuster S (2017) Modelling the host-pathogen interactions of macrophages and Candida albicans using game theory and dynamic optimization. J R Soc Interface 14(132):20170095

30. Dühring S, Germerodt S, Skerka C, Zipfel P, Dandekar T, Schuster S (2015) Host-pathogen interactions between the human innate immune system and Candida albicans-understanding and modeling defense and evasion strategies. Front Microbiol 6:625

31. Ebert D, Weisser WW (1997) Optimal killing for obligate killers: the evolution of life histories and virulence of semelparous parasites. Proc R Soc Lond Ser B Biol Sci 264(1384):985-991

32. Eckhoff PA, Wenger EA, Godfray HCJ, Burt A (2017) Impact of mosquito gene drive on malaria elimination in a computational model with explicit spatial and temporal dynamics. Proc Natl Acad Sci 114(2):E255-E264

33. Eftimie R, Gillard JJ, Cantrell DA (2016) Mathematical models for immunology: current state of the art and future research directions. Bull Math Biol 78(10):2091-2134

34. Ehrlich P (1913) Address in pathology, on chemiotherapy: delivered before the seventeenth international congress of medicine. Br Med J 2(2746):353-359

35. Ellner SP, Guckenheimer J (2011) Dynamic models in biology. Princeton University Press, Princeton

36. Eswarappa SM (2009) Location of pathogenic bacteria during persistent infections: insights from an analysis using game theory. PLoS One 4(4):e5383

37. Ewald J, Bartl M, Dandekar T, Kaleta C (2017) Optimality principles reveal a complex interplay of intermediate toxicity and kinetic efficiency in the regulation of prokaryotic metabolism. PLoS Comput Biol 13(2):e1005371

38. Ewald J, Bartl M, Kaleta C (2017) Deciphering the regulation of metabolism with dynamic optimization: an overview of recent advances. Biochem Soc Trans 45(4):1035-1043

39. Federico P, Gross LJ, Lenhart S, Ryan D (2012) Optimal control in individual-based models: implications from aggregated methods. Am Nat 181(1):64-77

40. Frohner IE, Bourgeois C, Yatsyk K, Majer O, Kuchler K (2009) Candida albicans cell surface superoxide dismutases degrade host-derived reactive oxygen species to escape innate immune surveillance. Mol Microbiol 71(1):240-252

41. Gao D, Lietman TM, Porco TC (2015) Antibiotic resistance as collateral damage: the tragedy of the commons in a two-disease setting. Math Biosci 263:121-132

42. Gardner M (1970) Mathematical games. Sci Am 222(6):132-140

43. Garg SK, Weinzimer SA, Tamborlane WV, Buckingham BA, Bode BW, Bailey TS, Brazg RL, Ilany J, Slover RH, Anderson SM et al (2017) Glucose outcomes with the in-home use of a hybrid closed-loop insulin delivery system in adolescents and adults with type 1 diabetes. Diabetes Technol Ther 19(3):155-163

44. Germerodt S, Bohl K, Lück A, Pande S, Schröter A, Kaleta C, Schuster S, Kost C (2016) Pervasive selection for cooperative cross-feeding in bacterial communities. PLoS Comput Biol 12(6): 1004986

45. Gilbert J, Blaser M, Caporaso J, Jansson J, Lynch S, Knight R (2018) Current understanding of the human microbiome. Nat Med 24(4):392-400

46. Gintis H (2000) Game theory evolving: a problem-centered introduction to modeling strategic behavior. Princeton University Press, Princeton

47. Görlich D, Escuela G, Gruenert G, Dittrich P, Ibrahim B (2014) Molecular codes through complex formation in a model of the human inner kinetochore. Biosemiotics 7(2):223-247

48. Gorochowski TE, Matyjaszkiewicz A, Todd T, Oak N, Kowalska K, Reid S, Tsaneva-Atanasova KT, Savery NJ, Grierson CS, Bernardo MD et al (2012) BSim: an agent-based tool for modeling bacterial populations in systems and synthetic biology. PloS One $7(8): \mathrm{e} 42790$

49. Gruenert G, Ibrahim B, Lenser T, Lohel M, Hinze T, Dittrich P (2010) Rule-based spatial modeling with diffusing, geometrically constrained molecules. BMC Bioinform 11(1):307

50. Gruenert G, Szymanski J, Holley J, Escuela G, Diem A, Ibrahim B, Adamatzky A, Gorecki J, Dittrich P (2013) Multi-scale modelling of computers made from excitable chemical droplets. IJUC 9(3-4):237-266

51. Haanstra JR, Gerding A, Dolga AM, Sorgdrager FJ, Buist-Homan M, Du Toit F, Faber KN, Holzhütter HG, Szöör B, Matthews KR et al (2017) Targeting pathogen metabolism without collateral damage to the host. Sci Rep 7:40406

52. Handel A, Liao LE, Beauchemin CA (2018) Progress and trends in mathematical modelling of influenza a virus infections. Curr Opin Syst Biol 12:30-36

53. Henze R, Dittrich P, Ibrahim B (2017) A dynamical model for activating and silencing the mitotic checkpoint. Sci Rep $7(1): 3865$

54. Henze R, Grünert G, Ibrahim B, Dittrich P (2019) Spatial rulebased simulations: the SRSim software. Springer, New York

55. Henze R, Huwald J, Mostajo N, Dittrich P, Ibrahim B (2015) Structural analysis of in silico mutant experiments of human inner-kinetochore structure. BioSystems 127:47-59

56. Henze R, Mu C, Puljiz M, Kamaleson N, Huwald J, Haslegrave J, di Fenizio PS, Parker D, Good C, Rowe JE et al (2019) 
Multi-scale stochastic organization-oriented coarse-graining exemplified on the human mitotic checkpoint. Sci Rep 9(1):3902

57. Hofbauer J, Sigmund K (1998) Evolutionary games and population dynamics. Cambridge University Press, Cambridge

58. Holen ØH, Johnstone RA (2004) The evolution of mimicry under constraints. Am Nat 164(5):598-613

59. Hummert S, Bohl K, Basanta D, Deutsch A, Werner S, Theißen G, Schroeter A, Schuster S (2014) Evolutionary game theory: cells as players. Mol BioSyst 10(12):3044-3065

60. Hummert S, Glock C, Lang SN, Hummert C, Skerka C, Zipfel PF, Germerodt S, Schuster S (2018) Playing 'hide-and-seek' with factor h: game-theoretical analysis of a single nucleotide polymorphism. J R Soc Interface 15(142):20170963

61. Hummert S, Hummert C, Schröter A, Hube B, Schuster S (2010) Game theoretical modelling of survival strategies of Candida albicans inside macrophages. J Theor Biol 264(2):312-318

62. Ibrahim B (2015) In silico spatial simulations reveal that MCC formation and excess BubR1 are required for tight inhibition of the anaphase-promoting complex. Mol BioSyst 11(11):2867-2877

63. Ibrahim B (2015) Spindle assembly checkpoint is sufficient for complete cdc20 sequestering in mitotic control. Comput Struct Biotechnol J 13:320-328

64. Ibrahim B (2015) Systems biology modeling of five pathways for regulation and potent inhibition of the anaphase-promoting complex (APC/C): Pivotal roles for MCC and BubR1. OMICS 19(5):294-305

65. Ibrahim B (2015) Toward a systems-level view of mitotic checkpoints. Prog Biophys Mol Biol 117(2-3):217-224

66. Ibrahim B (2017) A mathematical framework for kinetochoredriven activation feedback in the mitotic checkpoint. Bull Math Biol 79(5):1183-1200

67. Ibrahim B, Diekmann S, Schmitt E, Dittrich P (2008) In-silico modeling of the mitotic spindle assembly checkpoint. PLoS One 3(2): 1555

68. Ibrahim B, Dittrich P, Diekmann S, Schmitt E (2007) Stochastic effects in a compartmental model for mitotic checkpoint regulation. J Integr Bioinform 4(3):77-88

69. Ibrahim B, Dittrich P, Diekmann S, Schmitt E (2008) Mad2 binding is not sufficient for complete cdc20 sequestering in mitotic transition control (an in silico study). Biophys Chem 134(1-2):93-100

70. Ibrahim B, Henze R (2014) Active transport can greatly enhance cdc20: Mad2 formation. Int J Mol Sci 15(10):19074-19091

71. Ibrahim B, Henze R, Gruenert G, Egbert M, Huwald J, Dittrich P (2013) Spatial rule-based modeling: a method and its application to the human mitotic kinetochore. Cells 2(3):506-544

72. Javarone MA (2018) The host-pathogen game: an evolutionary approach to biological competitions. Front Phys 6:94

73. Klipp E, Heinrich R, Holzhütter HG (2002) Prediction of temporal gene expression: Metabolic optimization by re-distribution of enzyme activities. Eur J Biochem 269(22):5406-5413

74. Kreyssig P, Escuela G, Reynaert B, Veloz T, Ibrahim B, Dittrich $P$ (2012) Cycles and the qualitative evolution of chemical systems. PLoS One 7(10): 45772

75. Kreyssig P, Wozar C, Peter S, Veloz T, Ibrahim B, Dittrich $P$ (2014) Effects of small particle numbers on long-term behaviour in discrete biochemical systems. Bioinformatics 30(17):i475-i481

76. Kumar A (2014) An alternate pathophysiologic paradigm of sepsis and septic shock: implications for optimizing antimicrobial therapy. Virulence 5(1):80-97

77. Lang SN, Germerodt S, Glock C, Skerka C, Zipfel PF, Schuster S (2019) Molecular crypsis by pathogenic fungi using human factor h. a numerical model. PLoS One 14(2):e0212187
78. Lardon LA, Merkey BV, Martins S, Dötsch A, Picioreanu C, Kreft JU, Smets BF (2011) iDynoMiCS: next-generation individual-based modelling of biofilms. Environ Microbiol 13(9):2416-2434

79. Leach MD, Tyc KM, Brown AJP, Klipp E (2012) Modelling the regulation of thermal adaptation in Candida albicans, a major fungal pathogen of humans. PLoS One 7(3):1-14

80. Lenhart S, Workman JT (2007) Optimal control applied to biological models. Crc Press, Boca Raton

81. Lively CM, Dybdahl MF (2000) Parasite adaptation to locally common host genotypes. Nature 405(6787):679-681

82. Luke S, Cioffi-Revilla C, Panait L, Sullivan K, Balan G (2005) MASON: a multiagent simulation environment. Simulation 81(7):517-527

83. Magni L, Raimondo DM, Dalla Man C, De Nicolao G, Kovatchev B, Cobelli C (2009) Model predictive control of glucose concentration in type i diabetic patients: an in silico trial. Biomed Signal Process Control 4(4):338-346

84. Martinez MN, Papich MG, Drusano GL (2012) Dosing regimen matters: the importance of early intervention and rapid attainment of the pharmacokinetic/pharmacodynamic target. Antimicrob Agents Chemother 56(6):2795-2805

85. Matyjaszkiewicz A, Fiore G, Annunziata F, Grierson CS, Savery NJ, Marucci L, Bernardo MD (2017) BSim 2.0: an advanced agent-based cell simulator. ACS Synth Biol 6(10):1969-1972

86. McKenzie C (2011) Antibiotic dosing in critical illness. J Antimicrob Chemother 66(Suppl-2):ii25-ii31

87. Morozov A, Best A (2012) Predation on infected host promotes evolutionary branching of virulence and pathogens' biodiversity. J Theor Biol 307:29-36

88. Neumann G, Schuster S (2007) Continuous model for the rockscissors-paper game between bacteriocin producing bacteria. J Math Biol 54(6):815-846

89. Oremland M, Michels KR, Bettina AM, Lawrence C, Mehrad B, Laubenbacher R (2016) A computational model of invasive aspergillosis in the lung and the role of iron. BMC Syst Biol 10(1):34

90. Organization WH et al (2018) Mortality and global health estimates: Causes of death; projections for 2015-2030; projection of death rates

91. Osborne JM, Fletcher AG, Pitt-Francis JM, Maini PK, Gavaghan DJ (2017) Comparing individual-based approaches to modelling the self-organization of multicellular tissues. PLoS Comput Biol 13(2):e1005387

92. Osbourn A, Clarke B, Dow J, Daniels M (1991) Partial characterization of avenacinase from gaeumannomyces graminis var. avenae. Physiol Mol Plant Pathol 38(4):301-312

93. Pangburn MK, Müller-Eberhard HJ (1984) The alternative pathway of complement. Springer Semin Immunopathol 7(2-3):163-192

94. Pareja-Jaime Y, Roncero MIG, Ruiz-Roldán MC (2008) Tomatinase from Fusarium oxysporum $f$. sp. lycopersici is required for full virulence on tomato plants. Mol Plant Microbe Interact 21(6):728-736

95. Patel M, Raymond B, Bonsall MB, West SA (2019) Crystal toxins and the volunteer's dilemma in bacteria. J Evol Biol 32:310-319

96. Peer X, An G (2014) Agent-based model of fecal microbial transplant effect on bile acid metabolism on suppressing Clostridium difficile infection: an example of agent-based modeling of intestinal bacterial infection. J Pharmacokinet Pharmacodyn 41(5):493-507

97. Peña-Miller R, Lähnemann D, Schulenburg H, Ackermann M, Beardmore R (2012) Selecting against antibiotic-resistant 
pathogens: optimal treatments in the presence of commensal bacteria. Bull Math Biol 74(4):908-934

98. Perelson AS, Goldstein B, Rocklin S (1980) Optimal strategies in immunology III. the IgM-IgG switch. J Math Biol 10(3):209-256

99. Perelson AS, Mirmirani M, Oster GF (1976) Optimal strategies in immunology i. J Math Biol 3(3-4):325-367

100. Perelson AS, Mirmirani M, Oster GF (1978) Optimal strategies in immunology ii. J Math Biol 5(3):213-256

101. Peter S, Hölzer M, Lamkiewic K, di Fenizio PS, Al Hwaeer H, Marz M, Schuster S, Dittrich P, Ibrahim B (2019) Structure and hierarchy of influenza virus models revealed by reaction network analysis. Viruses 11(5):449

102. Pollmächer J, Figge MT (2014) Agent-based model of human alveoli predicts chemotactic signaling by epithelial cells during early Aspergillus fumigatus infection. PloS one 9(10):e111630

103. Pollmächer J, Figge MT (2015) Deciphering chemokine properties by a hybrid agent-based model of Aspergillus fumigatus infection in human alveoli. Front Microbiol 6:503

104. Pollmächer J, Timme S, Schuster S, Brakhage AA, Zipfel PF, Figge MT (2016) Deciphering the counterplay of Aspergillus fumigatus infection and host inflammation by evolutionary games on graphs. Sci Rep 6:27807

105. Porco TC, Gao D, Scott JC, Shim E, Enanoria WT, Galvani AP, Lietman TM (2012) When does overuse of antibiotics become a tragedy of the commons? PLoS One 7(12):e46505

106. Renaud F, De Meeüs T (1991) A simple model of host-parasite evolutionary relationships. parasitism: compromise or conflict? J Theor Biol 152(3):319-327

107. Richter H (2017) Dynamic landscape models of coevolutionary games. Biosystems 153-154:26-44

108. Savary S, Willocquet L, Pethybridge SJ, Esker P, McRoberts N, Nelson A (2019) The global burden of pathogens and pests on major food crops. Nat Ecol Evol 3(3):430-439

109. Schleicher J, Conrad T, Gustafsson M, Cedersund G, Guthke R, Linde J (2016) Facing the challenges of multiscale modelling of bacterial and fungal pathogen-host interactions. Brief Funct Genomics 16(2):57-69

110. Schluter J, Foster KR (2012) The evolution of mutualism in gut microbiota via host epithelial selection. PLoS Biol 10:e1001424

111. Schuster S, Ewald J, Dandekar T, Dühring S (2019) Optimizing defence, counter-defence and counter-counter defence in parasitic and trophic interactions-a modelling study. arXiv:1907.04820

112. Seal JB, Alverdy JC, Zaborina O, An G (2011) Agent-based dynamic knowledge representation of Pseudomonas aeruginosa virulence activation in the stressed gut: towards characterizing host-pathogen interactions in gut-derived sepsis. Theor Biol Med Modell 8(1):33

113. Sharomi O, Malik T (2017) Optimal control in epidemiology. Ann Oper Res 251(1-2):55-71

114. Shi ZZ, Wu CH, Ben-Arieh D (2014) Agent-based model: a surging tool to simulate infectious diseases in the immune system. Open J Model Simul 02(01):12-22

115. Shudo E, Iwasa Y (2001) Inducible defense against pathogens and parasites: optimal choice among multiple options. J Theor Biol 209(2):233-247

116. Shudo E, Iwasa Y (2004) Dynamic optimization of host defense, immune memory, and post-infection pathogen levels in mammals. J Theor Biol 228(1):17-29

117. Siettos CI, Russo L (2013) Mathematical modeling of infectious disease dynamics. Virulence 4(4):295-306

118. Soheilypour M, Mofrad MRK (2018) Agent-based modeling in molecular systems biology. BioEssays 40(7):e1800020

119. Speed MP, Ruxton GD (2010) Imperfect batesian mimicry and the conspicuousness costs of mimetic resemblance. Am Nat 176(1):E1-E14
120. Stengel RF, Ghigliazza R (2004) Stochastic optimal therapy for enhanced immune response. Math Biosci 191(2):123-142

121. Stengel RF, Ghigliazza R, Kulkarni N, Laplace O (2002) Optimal control of innate immune response. Opt Control Appl Methods 23(2):91-104

122. Stengel RF, Ghigliazza RM, Kulkarni NV (2002) Optimal enhancement of immune response. Bioinformatics 18(9):1227-1235

123. Stern JR, Olivas AD, Valuckaite V, Zaborina O, Alverdy JC, An G (2013) Agent-based model of epithelial host-pathogen interactions in anastomotic leak. J Surg Res 184(2):730-738

124. Tago D, Meyer DF (2016) Economic game theory to model the attenuation of virulence of an obligate intracellular bacterium. Front Cell Infect Microbiol 6:86

125. Tokarski C, Hummert S, Mech F, Figge MT, Germerodt S, Schroeter A, Schuster S (2012) Agent-based modeling approach of immune defense against spores of opportunistic human pathogenic fungi. Front Microbiol 3:129

126. Tschernyschkow S, Herda S, Gruenert G, Doering V, Görlich D, Hofmeister A, Hoischen C, Dittrich P, Diekmann S, Ibrahim B (2013) Rule-based modeling and simulations of the inner kinetochore structure. Prog Biophys Mol Biol 113(1):33-45

127. Tyc KM, Herwald SE, Hogan JA, Pierce JV, Klipp E, Kumamoto CA (2016) The game theory of Candida albicans colonization dynamics reveals host status-responsive gene expression. BMC Syst Biol 10(1):20

128. Tyc KM, Kühn C, Wilson D, Klipp E (2014) Assessing the advantage of morphological changes in Candida albicans: a game theoretical study. Front Microbiol 5:41

129. Tyson JJ, Chen KC, Novak B (2003) Sniffers, buzzers, toggles and blinkers: dynamics of regulatory and signaling pathways in the cell. Curr Opin Cell Biol 15(2):221-231

130. Tyson JJ, Novak B (2014) Control of cell growth, division and death: information processing in living cells. Interface focus 4(3):20130070

131. Ventola CL (2015) The antibiotic resistance crisis: part 1: causes and threats. Pharm Ther 40(4):277-283

132. Wilensky U (1999) Netlogo. http://ccl.northwestern.edu/netlogo/. Accessed 29 Apr 2019

133. Wiley RH (1994) Errors, exaggeration, and deception in animal communication. In: Behav Mech Evol Ecol. University of Chicago Press, pp 157-189

134. Williams RA, Timmis J, Qwarnstrom EE (2014) Computational models of the $\mathrm{nf}-\mathrm{kb}$ signalling pathway. Computation 2(4):131-158

135. Wu A, Ross D (2016) Evolutionary game between commensal and pathogenic microbes in intestinal microbiota. Games 7(3):26

136. Zipfel PF, Hallström T, Riesbeck K (2013) Human complement control and complement evasion by pathogenic microbes-tipping the balance. Mol Immunol 56(3):152-160

137. Zipfel PF, Skerka C (2009) Complement regulators and inhibitory proteins. Nat Rev Immunol 9(10):729-740

138. Zitelli G, Djouadi SM, Day JD (2015) Combining robust state estimation with nonlinear model predictive control to regulate the acute inflammatory response to pathogen. Math Biosci Eng 12:1127-1139

139. Zitzmann C, Kaderali L (2018) Mathematical analysis of viral replication dynamics and antiviral treatment strategies: from basic models to age-based multi-scale modeling. Front Microbiol 9:1546

Publisher's Note Springer Nature remains neutral with regard to jurisdictional claims in published maps and institutional affiliations. 